\title{
Publishing without being damned
}

A remarkable research report claims that an object spinning on a vertical axis weighs less if spinning clockwise (seen from above) than anticlockwise, but it may be too soon to cast off Newton's laws.

WHAT should a journal do when a contributor sends it a piece of research whose conclusions fly in the face of current beliefs? There are several responses, among which the exercise of studied scepticism is the most common. Contributors can be challenged almost indefinitely by increasingly zealous referees and may sometimes throw in the sponge. But what happens if the referees find nothing wrong, but are still unpersuaded of the conclusions? In a celebrated case in 1988, this journal published such a research report, following it by an on-site investigation and a denunciatory report, which concatenation of events was itself a cause of controversy.

In very different circumstances, Physical Review Letters, the American Institute of Physics' most eagerly read journal, has just dealt with such a contribution much more simply, merely by publishing it. No disrespect to any of those concerned, authors, referees or editors, is intended by the remark that the outcome is thoroughly unsatisfactory for the other interest-group vividly engaged in the publication process - the readers.

These are the circumstances. Two Japanese at the Tohoku University in Sendai, Hideo Hayasaka and Sakae Takeuchi, have carried out a simple experiment with a gyroscope (or, more exactly, with three gyroscopes): they have simply set it spinning and then weighed it, using a chemical balance (Phys. Rev. Lett. 63,$2701 ; 1989$ ). Some measure of the journal's embarrassment can be gleaned from the time spent in brooding on this research report, which was received for publication on 7 March 1988, revised by the authors by 9 August last year and published only at the beginning of the Christmas torpor.

The expectation is, of course, that the weight of a spinning gyroscope should be independent of its rotational speed or of whether or not it is spinning at all. What the authors find is that expectation is confirmed if the angular momentum vector of the rotation is directed upwards, against the direction of the Earth's gravitational field, or if the rotation viewed from above is anticlockwise. But - this is the surprise - if the angular momentum vector is directed downwards, towards the centre of the Earth, the weight is decreased.

The effect seems unambiguous, amounting to as much as $10 \mathrm{mg}$ in a total of $175 \mathrm{~g}$ (the weight of the heaviest gyro- scope), which is well within the sensitivity of the balance $(0.3 \mathrm{mg})$. The reduction of weight increases linearly with rotation speed and, for a given speed, is the same fraction of the weight. The balance measurements have been confirmed with a quite different electronic instrument in which deflections of a horizontal lever are compensated for by electromagnetic forces.

In all the measurements, the rotor of the gyroscope, housed in a metal cage, has been set spinning by means of a voltage amplifier, which is then disconnected. For the purposes of measurement, the gyroscopes have been placed within evacuated chambers sitting on one balance pan. In the measurements with the chemical balance, made of non-magnetic materials, the weight changes have been recorded both while the gyroscopes are shielded locally and then when the whole equipment is placed within a magnetically shielded room. Dutifully, but unsurprisingly, the authors have turned their spinning gyroscopes upside down, verifying in the process that the asymmetry they observe is simply a function of the direction of rotation, not of the orientation of the gyroscopes. The only obvious precaution against systematic errors not mentioned is that of swapping the gyroscopes from one balance pan to the other, but it is difficult to see why that should make a difference to the outcome.

Needless to say, there is no way in which the observations can be accommodated within conventional theories of mechanics, classical or otherwise. Indeed, the authors state laconically that "the experimental result cannot be explained by the usual theories". The best bet is some kind of spin-spin coupling - in this case between the rotation of the gyroscope and that of the Earth - such as would be allowed by some versions of Einstein's theory of gravitation, but the measurements are uncomfortably huge by those standards and, in any case, there is no reason why parallel spins should repel each other when antiparallel spins are indifferent to each other's presence.

So what is to be made of the result? Curiously, what might justly be considered the most radical announcement to touch basic physics since Einstein's time, the fifth force of the past few years notwithstanding, has so far occasioned very little comment. Those convinced that the general press is forever seeking to publi- cize sensations will remark that newspapers and other general media have shied away from a story that would have justified some delicious headlines. Why should that have been the case? Can it be that the scientific community itself is discomfited? Certainly this journal has found opinions of the work of Hayasaka and Takeuchi sparse in the past few weeks. (Even so, one courageous spirit promises a publishable opinion very shortly.)

The plain but conventional truth is that it is too soon to take a view. On that view, the results, correct or not, are interesting but inconclusive on their own. The next step will be to see whether they are confirmed or, alternatively, contradicted by independent measurements. Then, the argument goes, will be time enough to wonder what, if anything, they mean. Meanwhile, the small but zealous band of those devoted to the notion that suitably managed gyroscopes contain a recipe for the levitation of massive objects and even, perhaps, for perpetual motion can be counted on to fill the months ahead with endless noise. No doubt, theoreticians skilled in the ways of chirally asymmetric particles such as neutrinos will join in the fun.

That is why it would have been helpful if Hayasaka and Takeuchi had said a little more about their measurements. How, for example, did they come to be weighing a rotating gyroscope? "To confirm the reflection symmetry relating to the rotational motion of objects in the gravitational field of the Earth . . ." is what they say, but had they cause to believe that this point had not previously been tested? With all those spinning satellites in orbit about the Earth accumulating upwards kicks of acceleration whenever their spin axes coincide with the upward vertical?

There are also pernickety questions to ask about the data - why are the error bars for weight, described as "the fluctuation of weight changes", greater for clockwise than for anticlockwise rotations at the same speed? No doubt all that will in due course be made plain, but how will Hayasaka and Takeushi occupy themselves in the meantime? The common canard that the science establishment (of which Physical Review Letters is certainly a part) is forever suppressing uncomfortable truths has been shown to be false, but those who astonish us in ways like these have the first duty to check that what they have to say stands up.

John Maddox 